\title{
A Simple Algorithm To Distribute Optimally a Number of External Base Stations in a Microcellular Environment
}

\author{
Ramón Agustí Comes, Xavier Monfort Querol, Jordi Pérez Romero \\ Department of Signal Theory and Communications \\ Technical University of Catalonia (UPC) \\ C/ Gran Capità, s/n Campus Nord-Edifici D4 \\ 08034 Barcelona-Spain \\ Corresponding author: Ramón Agustí Comes \\ Phone:34-3-401 6781 \\ Fax: 34-3-401 7200 \\ E-mail: ramon@xaloc.upc.es, jordip@xaloc.upc.es
}

\begin{abstract}
A simple algorithm is proposed for the optimal distribution of a number of base stations in a microcellular environment. Criteria are defined in order to decide the combinations of antennas that should be tested, and a quality factor is proposed to compare them. Results are presented for a specific environment.
\end{abstract}

\section{INTRODUCTION}

One of the most important stages in the design of a mobile communications system consists in deciding a proper location for the base stations. There are many tools available to calculate the coverage of an antenna according to a propagation model, but it is also necessary to have some kind of tool making it possible to deal with this problem of location and so avoid a haphazard process of trial and error. However, little effort has been devoted to this issue in the literature published to date. The objective of this paper is to present a simple and fast algorithm that gives an initial approach to an optimal base station distribution that can be used as a starting point in the process of obtaining a good real distribution for a microcellular outdoor scenario consisting of a regular distribution of buildings into parallel and perpendicular streets with antennas located below roof-top level, monopole antennas being the type to be used.

\section{DEFINING AN OPTIMAL DISTRIBUTION OF BASE STATIONS}

In order to find the best distribution of base stations, given the number $\mathrm{N}$ of antennas to use and the characteristics of the environment, it is necessary to try different combinations of $\mathrm{N}$ positions of the antennas and compare them, so the first step is to define a quality parameter that allows this comparison process and which must be easy and fast to calculate.
A first possible criterion for deciding whether a distribution of base stations is better than another is to compare them according to the power level received at the different points: a higher level means that the combination is more suitable. One way to measure this characteristic is to calculate the average power level of the different points considered. Another important aspect to focus on is the sensitivity level $\mathrm{S}$ defined by the system, in other words, the minimum power level needed by the receiver to work properly. As a result, we must penalize the quality factor of a distribution if it has a large number of points below this level. For example, in the DECT system, for which the application was first designed, this level is $-83 \mathrm{dBm}$, although a margin to prevent possible fading should also be considered, thus making this level higher.

The above discussion leads us to define the quality factor as

$$
\mathrm{Q}=\overline{\mathrm{P}_{\mathrm{r}}}(1+\rho)
$$

where $\bar{P}_{r}$ is the average power level in $\mathrm{dBm}$ received in all the points considered, assumed to be negative, and

$$
\rho=\frac{N_{\text {points_below_sersitivity_level }}}{N_{\text {total_point s }}}
$$

Another issue to consider is the importance of reducing the number of tested combinations by assuming that many of them may be discarded without any test (for example those combinations with all the antennas placed together). The following aspects concerning this problem should be considered:

1.- Reducing the overlapping coverage area between base stations. This can be reached by avoiding as much as possible those combinations where two or more antennas are in a LOS (Line Of Sight) situation. 
2.- Providing homogeneity of coverage throughout the environment in order to avoid those combinations that would produce high levels in a certain region and at the same time very low levels in other places. In other words, we are looking for a power distribution with low standard deviation. This can be achieved by placing the antennas as far apart as possible.

3.- Reducing the number of antennas used to cover the whole region, always maintaining the characteristics of a microcellular environment. This can be done by increasing the region covered by a single antenna by placing it on a street corner: thus we ensure that more points have LOS to this antenna, which means better power levels. If we also consider that there will be little difference in coverage whether the antenna is placed at one point or another of the intersection, we will assume that the central point of the intersection will represent all of its points, so that only this point will be considered as a candidate to have an antenna.

\section{DESCRIPTION OF THE WORK ENVIRONMENT}

As mentioned above, the objective is to locate a number of antennas in a microcellular outdoor environment. Consequently, the Uni-Lund model described in [1] was used to calculate the coverage. Nevertheless, other proposed models could be used instead without invalidating the planning approach we propose. This model considers a regular distribution of buildings into parallel and perpendicular streets with antennas located below-roof-top level, and defines a different model for the LOS and the NLOS situations. The attenuations for these two cases are given in expressions (3),(7), being $x$ the distance between the antenna and the receiver in the LOS case and $y$ the distance between the receiver and the intersection for the NLOS case (see Figure 1).

$$
L_{1}(x)=C_{2} \cdot\left[L_{21}(x)^{4}+L_{12}(x)^{4}\right]^{\frac{1}{4}}
$$

where

$$
\begin{aligned}
& C_{L} \approx\left(\frac{4 \pi}{\lambda}\right)^{2} \\
& L_{L 1}(x)=x^{n 1}, \\
& L_{L 2}(x)=x^{n 1} \cdot\left(\frac{x}{x_{L}}\right)^{n 2-n 1}
\end{aligned}
$$

$\mathrm{n}_{1}=2.13, \mathrm{n}_{2}=4.35, \mathrm{C}=33.4 \mathrm{~dB}$ being experimental results $x_{L}=\frac{4 \pi h_{t} h_{r}}{\lambda}$ is the turning-point, where a change in attenuation's slope occurs, as a function of antenna heights

$$
L_{N}=\left[u\left(y-y_{1}\right)-u\left(y-y_{2}\right)\right] \cdot f\left(y_{2}\right) \cdot \frac{\log _{0}(y)-\log _{0}\left(y_{1}\right)}{\log _{\alpha}\left(y_{2}\right)-\log _{0}\left(y_{1}\right)}+u\left(y-y_{2}\right) \cdot f(y)
$$

where $u(y)$ is the step function and

$$
\begin{aligned}
& f(y)=10 \cdot \log _{10}\left(\frac{y}{y_{0}}\right)^{n} \\
& y_{0}=8.92 \cdot \varphi+1.7
\end{aligned}
$$

$$
\begin{aligned}
& y_{1}=10.7 \cdot \varphi+0.22 \cdot w d+2.99 \\
& y_{2}=0.62 \cdot w d+4.9 \\
& n=2.75-1.13 e^{-23.4 \cdot \varphi}
\end{aligned}
$$

In addition, a graphical interface is needed to introduce the environment characteristics. We worked with AutoCAD release 13 and developed an $\mathrm{ADS}$ (AutoCAD Development System) application to calculate the coverage and to carry out the process of locating the base stations. More information about ADS applications can be found in [2]. The region that we selected to carry out this process is the North Campus of the Technical University of Catalonia (UPC), which is a suitable environment for the Uni-Lund model.

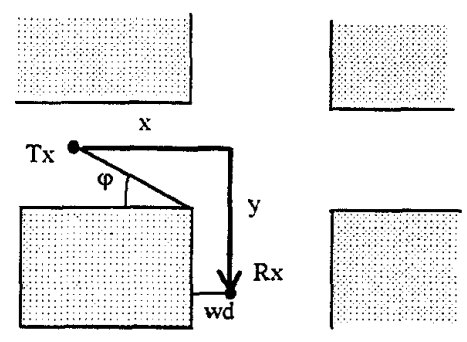

Figure1. Parameters for the Uni-Lund model.

\section{OPTIMAL BASE STATION DISTRIBUTION ALGORITHM}

The algorithm that we propose to find an optimal location for the base stations can be subdivided into the following steps:

1.- Data acquisition, using an AutoCAD drawing, of the characteristics of the environment. The drawing is then divided into small sections that we call resolution units, to each of which the corresponding height of the drawing is assigned. As a result of these two processes, we obtain a matrix that we call a height matrix, where each point $(i, j)$ has information about its corresponding height level.

2.- Detection of the intersections in the drawing. For this purpose, we make use of the height matrix. First of all, we transform this matrix into a binary one where each position $(i, j)$ has the value 0 if its height is lower than that of the antenna and 1 otherwise. We can consider this matrix as a binary image, made of pixels with values 0 and 1 . Then, all the technical methods used in digital image processing can be applied. In our case, we are interested in detecting the intersections of the streets that are present in the matrix and that have a very specific shape: a cross. As a result, we carried out a shape detection process by making use of a technique based on mathematical morphology used in non-linear digital image processing: dilation [3],[4]. This operation can be defined formally as follows: 


$$
\mathrm{f} \oplus \mathrm{B}=\max \mathrm{f}\left(\mathrm{x}+\mathrm{b}_{1}, \mathrm{y}+\mathrm{b}_{2}\right) \forall\left(\mathrm{b}_{1}, \mathrm{~b}_{2}\right) \in \mathrm{B}
$$

where $f(x, y)$ is the original image (the height matrix in our case) and $B$ is another matrix, called the structuring element, that contains the shape to be detected (a cross in our case) and can be considered as a mask.

Basically, this method consists in performing a series of superpositions of the structuring element and the matrix $f(x, y)$ by placing for each one the central point of $B$ at each point of $f(x, y)$ (this is the same as moving the mask B over the whole of the matrix $f(x, y))$. Then, for each superposition, the result will be the maximum value between all the points of $f(x, y)$ that are below the mask. In other words, for binary images, the result will be 0 if all the points below the mask are 0 , and 1 in all other cases. A simple example of this method is shown in Figure 2.

The final step, then, is to find the central point for each of the resulting groups of $0 \mathrm{~s}$ in the final matrix, which corresponds to the central point of each of the intersections in the original matrix. The result of the above process is a list of all the intersections in the environment that will be candidates to have an antenna. If a position is found that does not correspond exactly to a crossing, this does not pose any problem, as it will simply be an additional candidate to consider.

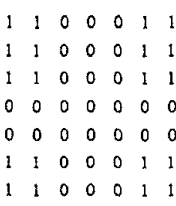

(a)

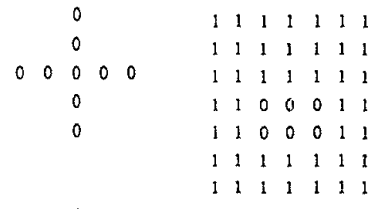

(c)
Figure 2. Matrices involved in the intersection detection process. (a) binary height matrix $f(x, y)$; (b) structuring element $B$ with the shape to detect (a cross); (c) Resulting matrix, where the intersection has been detected.

3.- Partition of the environment into $N$ regions, $N$ being the number of base stations to be distributed. In each region we will force the presence of just one antenna. This process allows us to separate the antennas as much as possible, in order to give a homogeneous coverage of the environment. We do this by dividing the whole environment into approximately equal rectangular regions. Some improvements could be made to this method by means of an adaptive process to carry out the partition when considering coverage values.

4.- Calculation of the coverage files for each candidate. This is the slowest process, but it must be said that it need only be done once if we only change $\mathrm{N}$ and not the environment or the antenna parameters (gain, height, etc.)

5.- Calculation of the overall coverage file (assuming as the value for the coverage the maximum level received from the various antennas) for each possible combination of $\mathrm{N}$ candidates, and the calculation of the quality factor defined in Equation 1. Finally, we choose the combination that gives us the highest value of $Q$.

\section{RESULTS FOR THE SELECTED ENVIRONMENT}

Figure 3 shows the results that we reached by using the process explained above in the UPC campus environment (approximately $340 \times 160 \mathrm{~m}$.) when we tried to locate $\mathrm{N}=3,4$ and 5 base stations, according to the DECT system, with monopoles working at $1.89 \mathrm{GHz}$, an ERP (Equivalent Radiated Power) of $24 \mathrm{dBm}$, an antenna height of $5 \mathrm{~m}$, and sensitivity levels of $\mathrm{S}=-83,-77,-75$ and $-71 \mathrm{dBm}$. More details of these results can be found in [5]. The quality factors obtained for these distributions are shown in Table 1.

\section{CONCLUSIONS}

A simple approach to determine an optimal distribution of $\mathrm{N}$ base stations in a microcellular environment has been proposed. First of all, a quality factor to compare different distributions has been defined, as well as some aspects to reduce the number of distributions to test. Afterwards, an algorithm has been proposed and it has been applied to a particular environment, showing the results obtained, that have been considered satisfactory as a first approximation in the process of determining the best locations for the base stations in a microcellular mobile communications system.

\section{REFERENCES}

[1] Börjeson, H., Bergljung, C., Olson, L.G., Karlsson, P., Öhrvik, S-O., "Using a Novel Model for Predicting Propagation Path Loss", COST 231 TD(93)86, University of Lund, Sweden, May 1993.

[2] Ransen, O., AutoCAD programming in $\mathrm{C} / \mathrm{C}++$, Ed. John Wiley \& Sons, Chichester, 1997.

[3] Serra, J.: Image Analysis and Mathematical Morphology, London: Academic Press, 1982.

[4] Dengler, J., Behrens, S., Desaga, J.F.: "Segmentations of Microcalcifications in Mammograms", in IEEE Transactions on Medical Imaging, Vol. 12, n.4, December 1993.

[5] Monfort, X., Pérez, J.: "Planificació d'un sistema DECT en un entorn de tipus campus", Projecte Final de Carrera, Radiocommunications Group, UPC-ETSETB, Barcelona, November 1997, Chapter 5. 


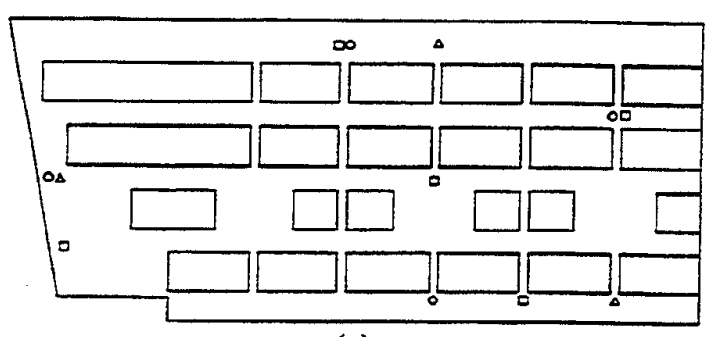

(a)

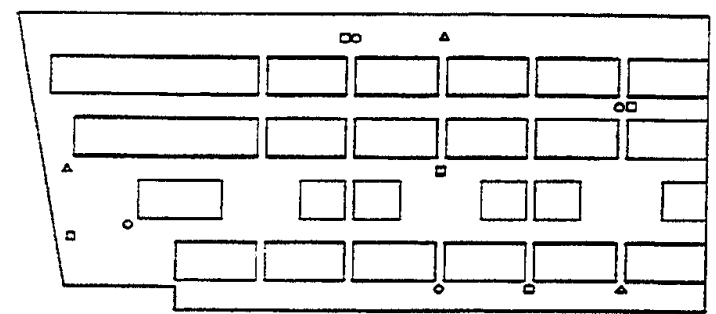

(b)

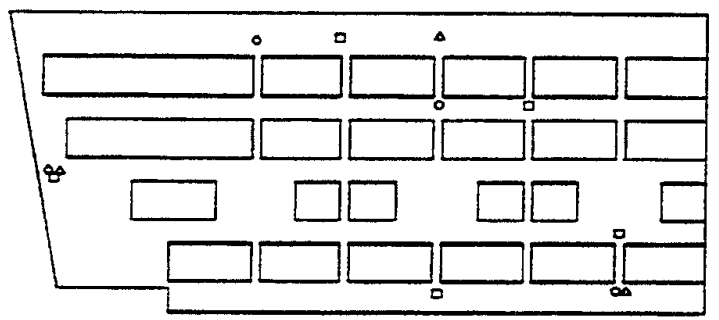

(c)

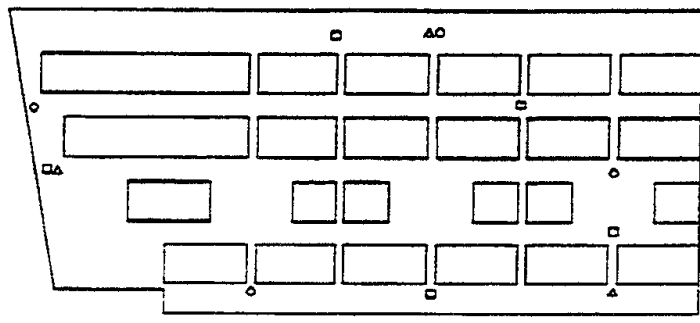

(d)

$\Delta$ Locations for the distribution of 3 antennas.

- Locations for the distribution of 4 antennas.

L Locations for the distribution of 5 antennas.

Figure 3. Aerial view of the 24 buildings of the UPC Campus Nord showing the optimal distributions of 3,4 and 5 antennas for sensitivity level (a)-83 dBm, (b) $-77 \mathrm{dBm}$, (c) $-75 \mathrm{dBm}$, (d) $-71 \mathrm{dBm}$

Table 1. Quality factors obtained in the analyzed distributions.

\begin{tabular}{|c|c|c|c|c|c|c|c|c|c|c|c|c|}
\hline $\mathrm{S}$ & \multicolumn{3}{|c|}{$-83 \mathrm{dBm}$} & \multicolumn{3}{c|}{$-77 \mathrm{dBm}$} & \multicolumn{3}{c|}{$-75 \mathrm{dBm}$} & \multicolumn{3}{c|}{$-71 \mathrm{dBm}$} \\
\hline $\mathrm{N}$ & $\mathrm{Q}(\mathrm{dBm})$ & $\mathrm{P}_{\mathrm{r}}(\mathrm{dBm})$ & $\rho(\%)$ & $\mathrm{Q}(\mathrm{dBm})$ & $\bar{P}_{\mathrm{r}}(\mathrm{dBm})$ & $\rho(\%)$ & $Q(\mathrm{dBm})$ & $\bar{P}_{\mathrm{r}}(\mathrm{dBm})$ & $\rho(\%)$ & $Q(\mathrm{dBm})$ & $\bar{P}_{\mathrm{r}}(\mathrm{dBm})$ & $\rho(\%)$ \\
\hline 3 & -53.68 & -53.57 & 0.21 & -55.02 & -53.57 & 3.08 & -56.02 & -53.57 & 4.59 & -57.75 & -53.57 & 7.81 \\
\hline 4 & -51.15 & -51.15 & 0 & -51.32 & -51.20 & 0.24 & -51.55 & -51.28 & 0.52 & -52.23 & -51.51 & 1.40 \\
\hline 5 & -49.03 & -49.03 & 0 & -49.08 & -49.03 & 0.10 & -49.11 & -49.11 & 0 & -49.12 & -49.11 & 0.02 \\
\hline
\end{tabular}

